\title{
GLADIS: GLobal Accretion Disk Instability Simulation
}

\author{
Agnieszka Janiuk* \\ Center for Theoretical Physics \\ Polish Academy of Sciences \\ Al. Lotnikow 32/46, 02-668 Warsaw, Poland \\ E-mail: agnes@cft.edu.pl
}

\begin{abstract}
I present the publicly available code GLADIS (GLobal Accretion Disk Instability Simulation) developed in my reserach group over the years 2002-2017. It can be freely downloaded and modified by the users via the link from the Astrophysics Source Code Library. The software computes time-dependent evolution of a black hole accretion disk, in one-dimensional, axisymmetric, vertically integrated scheme. The main applications are to explain the variability of accretion disks that can be subject to radiation-pressure instability. The phenomenon is relevant for fast variable microquasars, as well as for a class of changing-look AGN.
\end{abstract}

Multifrequency Behaviour of High Energy Cosmic Sources - XIII - MULTIF2019

3-8 June 2019

Palermo, Italy

${ }^{*}$ Speaker.
${ }^{\dagger}$ To the memory of my Mother 


\section{Introduction}

The accreting black holes observed in our Galaxy frequently exhibit the characteristic pattern of X-ray variablity. Their luminosity oscillations appear on short timescales (tens-hudreds seconds), and the regular outbursts typically have a profile with a slow rise, and fast decay of the X-ray flux in time. Two well-known microquasars, GRS 1915+105, and IGR J17091, in some states are presenting the variabity pattern called the 'heartbeat state' [1], because their oscillation shape resembles the electrocardiogram. It was also shown, that the timing properties of these heartbeats scale with the estimated mass of the black hole. In other sources, a substructure in between the main outbursts can be found, as they present a substructure and multiple peaks of varying amplitudes appear in their lightcurves. Nevertheless, in several sources, such as the black hole candidates GX 339-4, or XTE 1550-564, the non-linear variability was suggested based on the selected lighturves [2].

The lightcurves shape describes the time changes in radiation generated in the black hole accretion flow. This radiation is supposed to be driven by the energy dissipated through the viscous stresses within the relatively cold, soft X-ray emitting thin disk, and possibly transmitted to the hotter, hard X-ray emitting coronal flow. But even regardless of the details of the energy dissipation and radiative process, the traces of non-linear dynamics of the underlying physical process may be found by studying the observed lightcurves. As shown for instance by [3], the method of correlation dimension used in case of GRS 1915+105 for four of its temporal classes indicated that the underlying dynamical mechanism is a low-dimensional chaotic system. Also, the method of reccurrence analysis used by [4] revealed significant traces of nonlinear dynamics in three other sources: GX 339-4, XTE J1550-564 and GRO J1655-40, particularly in the disk-dominated soft state, as well as in the intermediate states at the rising and declining phase of the outburst.

\section{Non-linear processes in accretion disk}

Non-linear variability induced by the accretion disk instability, leads to periodic changes of brightness, and limit-cycle type of behaviour [5, 6].

\subsection{Local solutions}

The vertically averaged heating rate locally in the accretion disk, reads:

$$
Q_{+}=\frac{3}{2} \alpha P H \Omega_{\mathrm{K}},
$$

where $\alpha$ is the viscosity parameter as introduced by [7], $P$ is the pressure, $H$ is the disk halfthickness, and $\Omega_{K}$ is the Keplerian angular velocity. Cooling due to advection and radiative losses is given by:

$$
Q_{-}=\frac{4 \sigma_{B} T^{4}}{3 \kappa \Sigma}+F_{\text {tot }}\left(1-f_{\text {adv }}\right)
$$

where $\sigma_{B}$ is the Stefan-Boltzmann constant, $\kappa=0.34$ is the electron-scattering opacity, $T$ is the disk mid-plane temperature, and $\Sigma$ is the disk surface density. 
The closing equation for a stationary structure of the disk, is that for the total energy flux emitted from the surface unit area

$$
F_{\text {tot }}=\frac{3 G M \dot{M}}{8 \pi r^{3}} f(r)
$$

Here the global parameters are the mean mass accretion rate, $\dot{M}$, and black hole mass, $M$. The sero torrque boundary condition for $f(r)$ is typically used. The advection parameter in general is related the radial derivatives of density and temperature, but in a stationary model can ba adopted as a constant, $f_{\text {adv }}$, on the order of 0.3 . The accretion rate can be convieniently expressed in Eddington units:

$$
\dot{m}=\frac{\eta \dot{M} c^{2}}{L_{\mathrm{Edd}}}=\frac{\dot{M}}{\dot{M}_{\mathrm{Edd}}} ; \quad L_{\mathrm{Edd}}=\frac{4 \pi G M_{\mathrm{BH}} m_{\mathrm{p}} c}{\sigma_{\mathrm{T}}}=1.3 \cdot 10^{38}\left(M_{\mathrm{BH}} / M_{\odot}\right)
$$

The only non-vanishing stress-tensor component scales with pressure as:

$$
T_{r \phi}=-\alpha P
$$

where the total pressure $P$ is given by the gas and radiation components:

$$
P=P_{\mathrm{gas}}+P_{\mathrm{rad}}=\frac{\rho k_{B}}{m_{p}} T+\frac{4 \sigma_{B}}{3 c} T^{4}
$$

\subsection{Instabilities in accretion disks}

In accretion disks, we can have two types of thermal-viscous instabilities: (i) radiation pressure instability, $T \sim \Sigma^{-1 / 4}$ [8], and (ii) partial Hydrogen ionisation instability, driven by inverse dependence of opacity on temperature [9]. The first of these instabilities may lead to the short term limit cycle oscillations in black hole x-ray binaries (tens-hundreds seconds scales), or to a cyclic activity of quasars (scales of tens-thousands of years). The second type of instability has proven to induce the X-ray novae eruptions (scales of months-years), while it may also be related to the long-term activity cycles in AGN (scales of millions of years).

The localisation of the unstable regions in the disk shows two separate regions of the radiation pressure dominated zone, and the zone dominated by the partial Hydrogen ionisation [2]. In case of the radiation pressure instability, the disk can be stabilized by a strong jet/wind, which takes away some fraction of the locally dissipated energy flux. Also, by definition, the heating prescription will affect the disk behaviour, and the gass pressure dominated disk is completely stable. The influence of the companion star can also manifest itself as a stabilizing mechanism, when the mean mass inflow rate drops below the critical value for the instability onset. Finally, as shown in [10], the viscous fluctuations may partly stabilize the accretion disk.

\section{Code GLADIS}

The Code GLADIS (for GLobal Accredion Disk Instability Simulation) computes the dynamics of the radiation pressure unstable disk. There are two governing partial-differential equations to be solved numerically:

$$
\frac{\partial \Sigma}{\partial t}=\frac{1}{r} \frac{\partial}{\partial r}\left(3 r^{1 / 2} \frac{\partial}{\partial r}\left(r^{1 / 2} v \Sigma\right)\right)
$$


and

$$
\frac{\partial \ln T}{\partial t}+v_{r} \frac{\partial \ln T}{\partial r}=\frac{4-3 \beta}{12-10.5 \beta}\left(\frac{\partial \ln \Sigma}{\partial t}-\frac{\partial \ln H}{\partial t}+v_{r} \frac{\partial \ln \Sigma}{\partial r}\right)+\frac{Q_{+}-Q_{-}}{(12-10.5 \beta) P H}
$$

The derivation of above formula can be found e.g. in [11].

GLADIS computes the time-dependent evolution of a black hole accretion disk, in 1 dimensional, axisymmetric, vertically integrated scheme. The code solves the above equations for surface density and temperature evolution, i.e. given by viscous diffussion and energy conservation.

The code has been indexed in the Astrophysics Source Code Library http://ascl.net/1812.002 and ADS database (2018ascl.soft12002J) The package (tar.gz) with the source code is available at http://www.cft.edu.pl/astrofizyka/ under tab Numerics. The User can download the code sources written in $\mathrm{C} / \mathrm{C}++$, a sample makefile, the macros for initial configuration, and a running script. All the sourecs are available for download from the website of the Astrophysics Group at CTP PAS (see Figure 1).

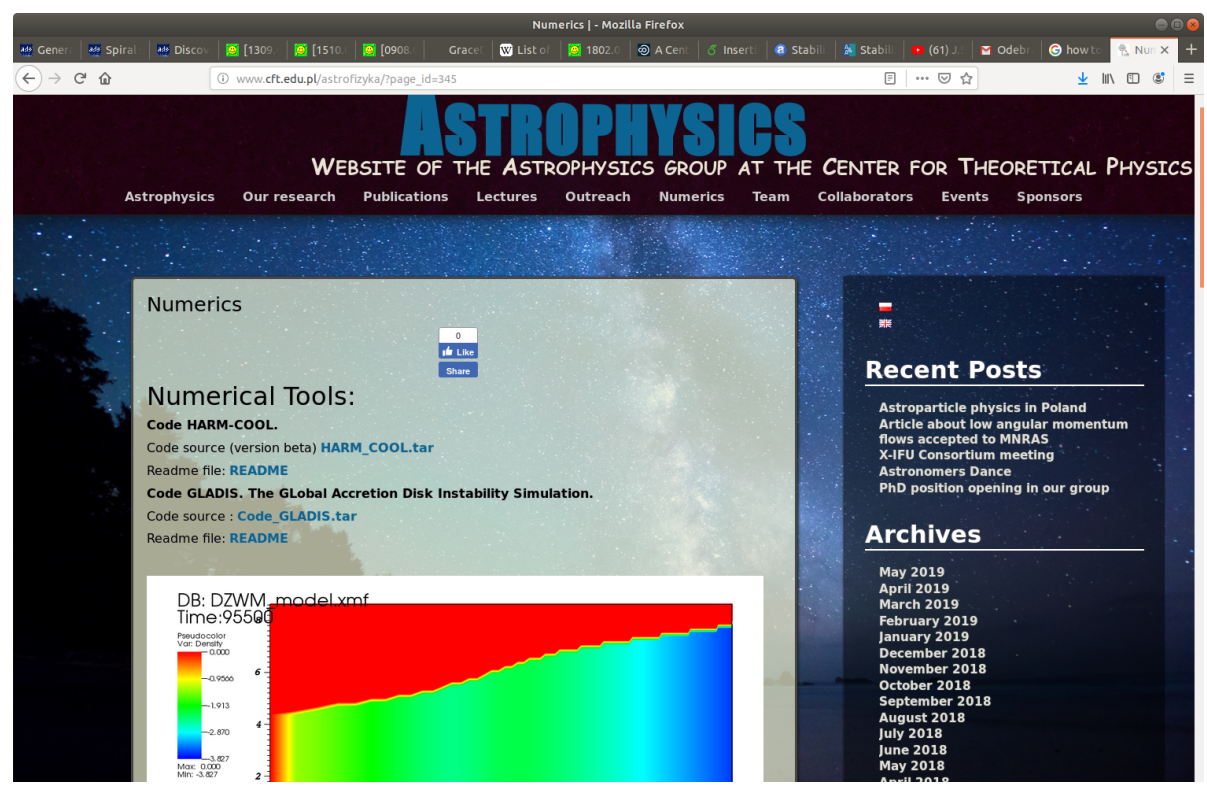

Figure 1: Website to download the code GLADIS

The initial conditions assume the stationary disk with a solution numerically integrated with Newton-Raphson scheme for the density, mid-plane temperature, and thickness profiles. The latter is taken from the hydrosytatic equilibrium condition. The time dependent simulation is run by the Eulerian code, with the $4^{\text {th }}$ order Runge-Kutta and Predictor-Corrector methods. The code is parallelized with MPI technique and can be run on multiple processors. The model parameters are stored in the configuration file dysk-zwm.ini. Their values refer to the physical setup as in [12], [13], and [10]. The code stores the output in various formats: ASCII and HDF5. Their content is described in detail in the README manual.

\subsection{Exemplary results for the set of parameters reproducing the microquasar IGR J17091}

We run the sample simulation with the set of parameters that represent microquasar IGR J17091-3624. In this source, the black hole mass is of $M=6 M_{\odot}$, and accretion rate estimated 
on $\dot{M}=0.86-2.21 \times 10^{-8} M_{\odot} / y r$. We use the upper limit value which corresponds to $\dot{m}=0.1$ in Eddington units. The adopted viscosity parameter is $\alpha=0.1$, and the outer radius of the grid was located at $R_{\text {out }}=1000 R_{\text {Schw }}$. We also prescribe a moderately stron wind outflow, with the normalisation $A=15$.

In Figure 2 we show a model lightcurve produced with the simulation using GLADIS. We also show the evolution of the surface density and disk temperature at the inner radius, $r=3.01 R_{\text {Schw }}$. It follows the loop around the unstable branch of the stability curve.

The integrated variablity pattern reproduces the heartbeat oscillation as observed in diskdominated states of the microquasar IGR J17091 (see details in [14]). As we have shown, the wind launched from the accretion disk and the heartbeat oscillations manifest actively their role in this source. The amplitudes of quasi-periodic flares are anti-correlated with the strenght of wind outflow. Moreover, the wind may help to partially or even completely stabilize the heartbeat during the soft state, i.e. the oscillations cease, when a stron wind is detected. We found that the constraints on the wind outflow rate from Chandra data in the no-heartbeat state give the mass loss rate in the wind $\dot{M}_{\text {wind }}=3-4.7 \times 10^{17} \mathrm{~g} \mathrm{~s}^{-1}$, while in the heartbeat state the wind mass loss is ten times smaller. The CLOUDY photoionisation code results are consistent with the wind strength and spatial dimension of the wind launching region determined from the GLADIS model.
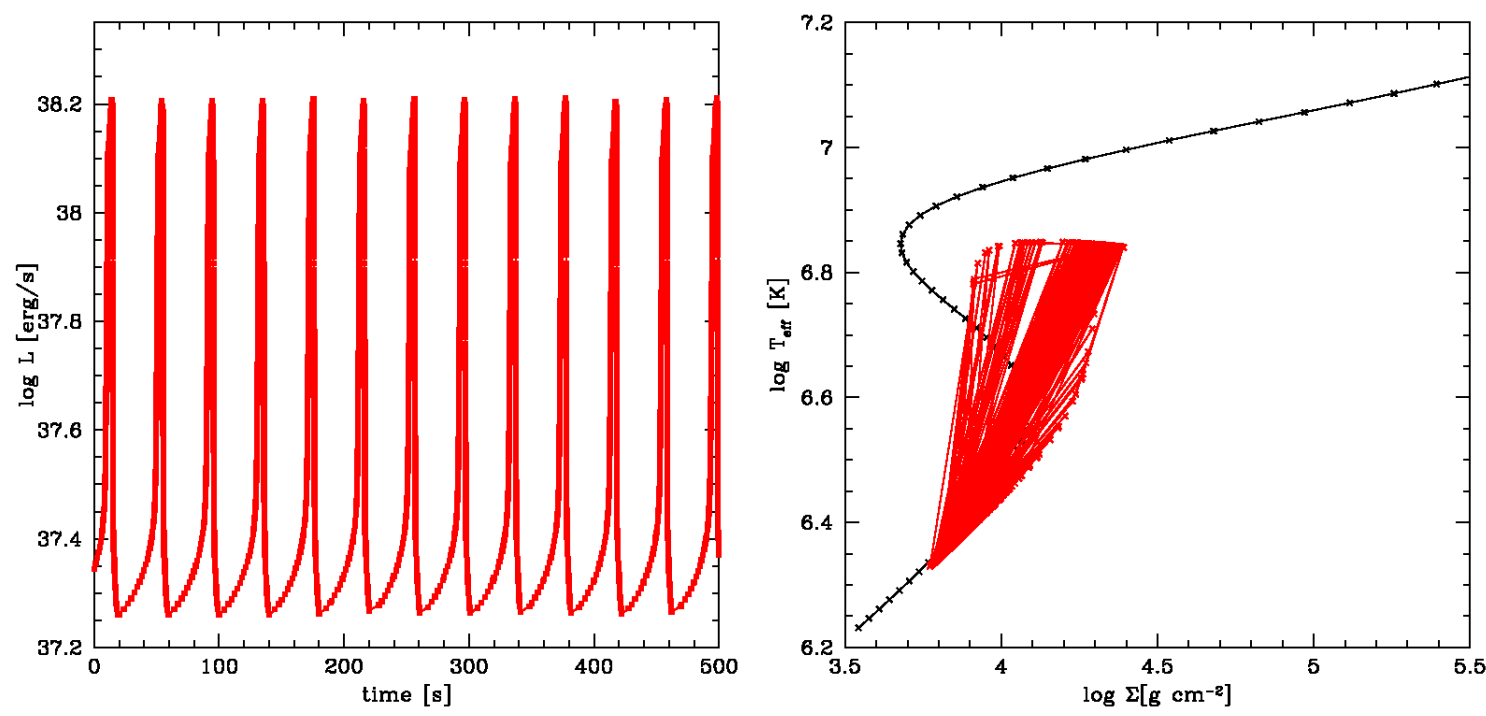

Figure 2: Left: Sample lightcurve simulated with the code GLADIS. Right: evolution of surface density and disk temperature at the inner radius. Parameters of the simulation refer to the source IGR 17091.

\section{Discussion of possible applications of the radiation pressure instability and its modeling with the code GLADIS}

\subsection{Viscosity fluctuations}

Another feature implemented in the GLADIS are the propagating viscosity fluctuations. The idea has been introduced by [15] to interpret the nature of accreting sources that exhibit a stochastic 
variability. Such kind of behaviour may naturally arise through viscous fluctuations in a turbulent disk. With this option, the viscosity in accretion disk is no longer a constant, but is a function of radius and time, as given by:

$$
\begin{gathered}
\alpha(r, t)=\alpha_{0}[1+\beta(r, t)] \\
\beta_{n}=b_{0} u_{n}=b_{0}\left(-0.5 u_{n-1}+R A N_{n}\right)
\end{gathered}
$$

We have shown that the above viscosity flickering stabilizes the disk oscillations induced by the radiation pressure [10]. The amplitude and period of flares decrease with the $b_{0}$ parameter.

\subsection{Modified stress tensor}

The simulations made with the code GLADIS have been used also as a new method for the black hole mass determination. In particular, the method was applied to the source HLX-1 [16]. This hyperluminous X-ray source $\left(10^{42} \mathrm{erg} \mathrm{s}^{-1}\right)$ near the spiral galaxy ESO $243-49$ underwent recurrent outbursts within a period of about 400 days, is possibly the best candidate for an intermediate mass black hole (IMBH).The timescales and amplitudes of these outbursts are consistent with the evolution of the radiation-pressure unstable accretion flow, if we account for the strong global magnetic field which can stabilize the disk. The parameter $\mu$ introduced to scale the strength of the radiation pressure contribution to the stress tensor as:

$$
T_{\mathrm{r} \phi}=\alpha P^{\mu} P_{\mathrm{gas}}^{1-\mu}
$$

can give an effective prescription for the magnetic field [17].

We found, that with $\mu=0.54$ the source HLX-1 fits in the mass-scale relation, spanning several orders of magnitude in the black hole mass range, from the two known microquasars, up to the Giga-Hertz Peak Spectrum radio sources [18]. Other best-fit parameters for this source are its black hole mass $M_{B H}=1.5 \times 10^{5} M_{\odot}$, and accretion rate of $\dot{m}=0.09-0.18$ of Eddington rate.

Finally, with implementation of the code GLADIS, we studied the influence on the disk stabilisation made by changes of opacities due to the absorption on heavy atoms. It is supposed that they have a local influence on the accretion disk stability, but this effect is very sensitive to the black hole mass. Our global simulations, with a range of $\mu$-paramater values for the stress tensor, have shown that the limit cycle oscillations appear. They are suffering some disturbances from the Iron Opacity Bump, but nevertheless are still expected in AGN over a wide range of black hole mass [19].

\section{Summary}

- We have shown that global time-dependent evolution of the accretion disk on viscous timescale leads to the regular limit-cycle oscillations for $T_{r \phi} \propto \alpha P$, even at moderate $\dot{m}$.

- Microquasar IGR J17091 was studied with respect to its unique properties, and it is the second known source (after GRS 1915+105) that proves the limit-cycle oscillations. Other sources, such as GX 339-4, and XTE J155, also show significant tracers of non-linear dynamics. Radiation pressure instability is universal across the $\mathrm{BH}$ mass-scale. 
- Code GLADIS is now available for the community to download and use. By mens of this tool, the user may search for the limit-cycle periodicities in the astrophysics sources.

- The most general version of the code setup allows for the wind parametrisation, and also $\alpha$-flickering propagations. Recently the code has been used in a number of applications, published in the specialist literature.

Acknowledgements We acknowledge partial support from grant DEC-2016/23/B/ST9/03114 awarded by the Polish National Science Center.

\section{References}

[1] D. Altamirano, T. Belloni, M. Linares, et al., The Faint "Heartbeats" of IGR J17091-3624: An Exceptional Black Hole Candidate, ApJL 74217 (2011)

[2] A. Janiuk, B. Czerny, On different types of instabilities in black hole accretion discs: implications for $X$-ray binaries and active galactic nuclei, MNRAS, 4142186 (2011)

[3] R. Misra, K.P. Harikrishnan, B. Mukhopadhyay, G. Ambika, A.K. Kembhavi, The Chaotic Behavior of the Black Hole System GRS 1915+105, ApJ 609313 (2004)

[4] P. Sukova, A. Janiuk, M. Grzedzielski, Chaotic and stochastic processes in the accretion flows of the black hole X-ray binaries revealed by recurrence analysis, A\&A 586143 (2016)

[5] F. Honma, R. Matsumoto, S. Kato, Nonlinear Oscillations of Thermally Unstable Slim Accretion Disks around a Neutron Star or a Black Hole PASJ, 43147 (1991)

[6] E. Szuszkiewicz, J. Miller, Limit-Cycle Behaviour of Thermally Unstable Accretion Flows on to Black Holes, MNRAS 888298 (1998)

[7] N.I. Shakura, R.A. Sunyaev, Black holes in binary systems. Observational appearance., A\&A 24337 (1973)

[8] A.P. Lightman, D.M. Eardley, Black Holes in Binary Systems: Instability of Disk Accretion, ApJ 187 L1 (1974)

[9] J. Smak, Accretion in cataclysmic binaries. IV. Accretion disks in dwarf novae., Acta Astron. 34161 (1984)

[10] A. Janiuk, R. Misra, Stabilization of radiation pressure dominated accretion disks through viscous fluctuations, A\&A $\mathbf{5 4 0} 114$ (2012)

[11] B. Paczynski, G. Bisnovatyi-Kogan, A Model of a Thin Accretion Disk around a Black Hole, Acta Astron. 31283 (1981)

[12] A. Janiuk, B. Czerny, Time-delays between the soft and hard X-ray bands in GRS 1915+105, MNRAS 356205 (2005)

[13] A. Janiuk, B. Czerny, Accreting corona model of the X-ray variability in soft state X-ray binaries and active galactic nuclei, A\&A 466793 (2007)

[14] A. Janiuk, M. Grzedzielski, F. Capitanio, S. Bianchi, Interplay between heartbeat oscillations and wind outflow in microquasar IGR J17091-3624, A\&A 57492 (2015)

[15] Y.E. Lyubarskii, Flicker noise in accretion discs, MNRAS, 292679 (1997) 
[16] Q. Wu, B. Czerny, M. Grzedzielski, et al., The Universal âĂIJHeartbeatâĂ I Oscillations in Black Hole Systems Across the Mass-scale, ApJ 83379 (2016)

[17] M. Grzedzielski, A., Janiuk, B. Czerny, Q. Wu, Modified viscosity in accretion disks. Application to Galactic black hole binaries, intermediate mass black holes, and active galactic nuclei A\&A $\mathbf{6 0 3} 110$ (2017a)

[18] B. Czerny, A. Siemiginowska, A. Janiuk, et al., Accretion Disk Model of Short-Timescale Intermittent Activity in Young Radio Sources, ApJ 698840 (2009)

[19] M. Grzedzielski, A., Janiuk, B. Czerny, Local Stability and Global Instability in Iron-opaque Disks ApJ 84520 (2017b)

\section{DISCUSSION}

GALINA LIPUNOVA: Can the code be changed if one wants to account for a different vertical structure?

AGNIESZKA JANIUK: The GLADIS code is vertically averaged. Nevertheless, the vertical structure is taken into account via the averaging coefficients. They are defined as the ratio between the vertically integrated density, pressure, and radiation flux, with respect to their equatorial values times the disk thickness (see Eqs. 16-18 in Janiuk et al. 2002). The coefficients were computed from the model of vertical structure that we had, but they might be verified with a different model and then changed in the GLADIS code. 